\title{
The Formation and Expression of Community Consciousness in the Pattern of Internet*
}

\author{
Kun Wang \\ Northwest Minzu University \\ Lanzhou, China
}

\begin{abstract}
This paper focuses on the relationship between the communication media and the convergence of community consciousness. First, it collated how the media constructed the imagination and emotion of the community from the oral communication to the electronic communication; secondly, in combination with the "The Emperor goes out" event, from the two aspects of field and discourse expression, it explored the role in the formation of the subject behavior dominated by the consciousness of the Internet space community; and finally, this paper made a reflection on the view of communication cognition of media tool theory from the academic level, and considered that the prosperity of media technology had changed its identity from "outsider" to architect and actor in the trend of thought and action of the community.
\end{abstract}

Keywords-community consciousness; media technology; "The Emperor bar" expedition; actors

\section{INTRODUCTION}

The so-called community refers to groups and organizations of different levels formed by common characteristics in society, ranging from small family to the whole humanity. The national community refers to the national community which is based on the state and exists in the macro narrative context. The community consciousness is the basic identity of the spirit and consciousness which is based on the concept of state. From the perspective of the national community, they are the basic identity of the internal members to the cultural community constructed in people's mind and collective memory through common historical and political planning. From the point of view of the occurrence process, the consciousness of national community and nationalism "are actually a changing relationship between the self and the other, and this changing relationship constantly reshapes their own identity" Therefore, the consciousness of national community is a kind of nationalism of the concept of state, and a kind of cognition and recognition of the relationship between the national members and the country, even the motherland. Its formation is to solve the fundamental problem of "who I am, where I come from", and also to clearly distinguish the relationship between me (US) and him (them). The formation of this ideology based on interests of state-nation is attributed to the concept of natural geography formed for a long time, and also to the common cultural tradition formed in the process

*Fund: It is a phased achievement of the Central University Project 31920190211) of cultural movement for a long time. This kind of consciousness tends to present the emotional representation through the stimulation of external events, and then plays the role of strengthening identity in self internalization.

In the process of human social evolution, technology has always been an important factor affecting social change. From the perspective of history, the breakthrough of key technologies and the structure of new technologies will bring about the transformation of social structure and even human culture. By breaking the limitations of time and space in the original production and life, a new life experience is formed. As an increasingly important technology in modern technology, information technology plays an increasingly important role in people's production, life, and concept shaping, and even the survival and development of the statenation are playing an increasingly important role. From the perspective of the evolution of communication technology and media, human beings have roughly experienced three stages: oral, text printing and electronic communication. In each stage, the media has a profound impact on the cognitive development of the nation-state and even the world.

In order to survive in the natural environment, to satisfy the psychology of calling and knowing, and to facilitate cooperation, ancient ancestors created language. Through language, symbolic information flows back and forth faceto-face, through expression behavior and information communication, the mutual understanding and cooperation between individuals are deepened, the way of common life and production is formed in a certain region, the concept of "tribe" described by McLuhan is constructed, a "tribal" consciousness of "we" is also formed. Although viewed from the perspective of time and space, this kind of communication seems to be inefficient, and the oral media has contributed to the emergence of the relationship between people, promoting the social community of "tribe", and laying the foundation for the formation of the concept of community.

Character, an external medium symbol, not only solves the limitation of time and space to language, but also promotes human communication to be rational. Words provide the meaning of entity symbols for language, memory as a solid entity, and provide the basic conditions for the formation of the concept of "collective consciousness" and the inheritance of collective culture. Especially after the birth of printing media, the national language and characters can 
be unified. As Anderson discussed in the community of imagination, printing technology transcends the oral face-toface way. Through printing products (books, especially newspapers and magazines), it can arouse people's memories about the "common" of regions and collectives, so as to promote a certain group to achieve the distinction between the other and form the imagination of national community about borders and culture. It is worth noting that the imagination of this community is not fiction or fabrication, but a kind of "social fact" defined by Durkheim from the perspective of social psychology, which has the binding force of "obedience" to its members and further strengthens the community consciousness. What's more, it is in this participation in the imagination of community psychology, emotion and ideology that the nation-state can be constructed in the world.

In the stage of electronic communication, the media represented by TV broke through the barrier of local self isolation and the boundary of physical time\& space, extended human body senses, and made people intuitively feel the imaginary "we". Different from the two kinds of media mentioned above, the characteristic of TV media scene is not only to reduce the personal and cordial feelings between individuals and groups, but also to promote people to connect the media reality and social reality, and to further promote people's cognition, identification and centripetal force to the cultural community through the stimulation of emotion and mood .In the new information revolution era, Internet media, with its leading role, is changing the way people communicate and connect in the world. Through the Internet, individuals can realize the meaning exchange, experience sharing, cultural symbiosis and emotional expression from regional absence to virtual "presence". The communication formed by the media's further breaking through the boundaries of time and space is accompanied by a deeper understanding of "we". Therefore, network media not only expands the scope of social communication, but also provides individuals with a sense of belonging beyond blood and geography, and constructs a broad space for the production, circulation and consumption of national consciousness and behavior to break through the boundaries of time and space and interact with each other.

\section{INTERNET: A NEW FIELD OF COMMUNITY CONSCIOUSNESS FORMATION}

Marx thought that consciousness is "due to the need, due to the urgent need to communicate with others." The reason for the demand is closely related to people's common activities in a certain social relationship. The continuous evolution of media expands the social network and enriches the categories of common activities, which leads to people's urgent need for communication. Especially in the 1990s, with the rapid expansion of the Internet in the world, digital technology has constructed a different space of human life cyberspace, and then came into being a network society with dual attributes of reality and virtual. In this new space and new form of society, the growth of national community consciousness has found unprecedented soil.
First of all, the interaction of free expression and information sharing of network provides an ideal space for the national community consciousness. The empowering nature of the Internet enables social individuals to have the right of speech expression to a certain extent, thus arousing the enthusiasm of people's expression, meeting the urgency of people's communication to a certain extent, and providing preconditions for the formation of national community consciousness. According to the Internet development report, by the end of December 2017, China's Internet users had reached 772 million, a year-on-year increase of 2.6\%.Among them, the use rate of We-chat friends circle users and QQ space users is $87.3 \%$ and $64 \%$ respectively; the use rate of micro-blog continues to grow, reaching $40.9 \%$; the use of Douban, Tianya community and other forums has also significantly improved. What these data show is that the audience's desire for free expression and some psychological or social needs are being met through the continuous release of social discourse. At the same time, it is also seen that the network circle group as a platform for information sharing and interaction has become the best choice for people's discourse release and consciousness condensation. People are free to show themselves and express their opinions on the platform of "presence of others". At the same time, they have reached a consensus on the significance of others' presence and interaction with others. As the sociologist Granowort analyzed in the theory of "weak joint and several advantages", because of the diversity of information and multi-dimensional views, the individual with weak link is more conducive to the formation of a complete and multi angle understanding of the problem, and also to the construction of an objective and rational concept of national community.

Since the 1990s, network media, especially circle communication, have been building and converging the consciousness of national community. In 1998 Indonesia's Anti-China incident, Chinese youth groups took the network community as the front to protest fiercely, which caused a strong public opinion at home and abroad. Then, college students as the main group, through the network to create "powerful country forum", "iron blood community", "April network" and other forums and websites, protest and denounce every violation of the country and compatriots. With the national and compatriot events constantly presented in front of the public by the network media, the community consciousness is constantly formed and converged, and the emotional expression of the country is constantly stimulated. At the same time, with the help of the network space, the public can realize the collective behaviors that are difficult to form in real life in the virtual space. Under this background, in 2003, Li Mujin put forward a new concept of "network nationalism" in an article published in the International Herald Leader, which combines the characteristics of the times, that is, "taking the network as the platform, making patriotic remarks and opposing narrow nationalism; taking the network as the" base area ", gathering people of the same aspiration and taking concrete actions against it".

The "emperor bar" expedition in 2016 was initiated in a community of internet fans actively expressed by the public, 
spread among the Internet circle groups, and combined with effective organizations, created a historical peak of influence of nationalism events dominated by community consciousness in recent years. It should be noted that the participants are different from the past, which is what a large number of media and public opinion call "little pink". Most of them are post-90s generation. They usually don't care much about politics, but they are very concerned about the dynamic of entertainment circle and online literature. They like star idols and spontaneously participate in the online nationalist movement. " In this event, "the identification of consumers and the highly organized\& ritualized collective actions that often occur in the online community are the most fundamental basis for these young netizens to be able to mobilize and unify their actions in an instant." For the transformation of the concept of idols and the state, some scholars think that "these nationalists with little pink as the main body have translated the way of chasing stars to the state.". In the face of national state doubt, growing up in the background of patriotism education, they use the same psychology to transform the national state's personal idols, "to construct the state as a 'transitional object' between their internal reality and external reality, to realize the communication with the external and the construction of their own identity."

Secondly, the network circle communication mode and viral transmission mechanism catalyze the large-scale generation of national community consciousness. Different individuals enter into the circle group because of their common interests or benefits. Through free communication and exchange of views, it is possible to form self and mutual identity and build mutual trust. Under the long-term interaction, the members of the circle group gradually form a common concept and values, and further strengthen the cohesion and stability of the circle group. At the same time, each network circle group is not completely closed, each member exists in different circle groups with different needs, which forms the communication form of circle group and circle group nesting and linking each other. In the circle group communication, firstly, the information in a circle group spreads from the opinion core to the edge in a multidirectional and disorderly way, then from the edge to the opinion center, and then from the center to the edge. This kind of communication mode of repeated circulation "follows the existing interpersonal relationship of members, forms the mass gathering and mobilization of points, lines and surfaces in the way of snowball", achieves the group consensus through the repeated information exchange and interaction within and between the circle groups, and plays a strong catalytic role in the manifestation of the national community consciousness in a short time and on a large scale.

Since the middle and late 1990s, the impact and sensitivity of foreign affairs have pushed the thought of national community to an unprecedented state of development. From the galaxy incident in 1993, China's bid to host the Olympic Games in 2000 failed, in 2008, the Beijing Olympic torch encountered protests from dissidents in France, to the Diaoyu Island incident in 2012, the mass media linked the incident with the people for the first time, triggering the whole society's voice of opposition and protest. Since the beginning of the new century, the events of foreign nationalism have frequently aroused the emotions and actions of the national community. It can be said that the large-scale collective action led by community consciousness triggered by the event benefits from the "informed" function of mass media and Internet media, which is closely connected with the participation of media. Let's look at the events of the "emperor bar" expedition over the years - the incident of Catherine Yang, the beating of a pregnant woman by members of the Korean men's League, the incident of Zhou Ziyu and the incident of independence of Hong Kong, the Chinese University of Hong Kong. We will find that, with the penetration of media into different dimensions of the country and individuals, the types of emotion and action events triggered by the national community are no longer limited to political and economic events, but more star and entertainment events. The interest, curiosity and emotional resonance of these events all meet the basic requirements of viral communication. In the communication mode of repeated interaction, the consciousness of national community is urged and made into action.

Once again, delving into the "Emperor bar Expedition", the whole process of participants from mobilization to consensus and then to concerted action follows the abovementioned communication mode and transmission mechanism. First of all, the participants' cognition of individual identity is a barman with tens of millions of attention. They form a certain degree of aggregation and interaction in daily communication, and cultivate a relatively stable group of emotions. Secondly, the theme of "expedition" involves the hallowed symbol of nation-state and motherland, arousing the consciousness of "NationState" identity and their sense of justice and responsibility. After that, opinion leaders gather daily loose views and different identity emotions through their strong personal views and infectious language, and form collective excitement through the repeated interaction of "center-edgecenter" of discourse, thus achieving a situation of "one hundred responses" in a short time. Since then, "Emperor bar" has launched several campaigns with similar action logic, and we can find that the action process of participants connected by weak ties presents the following common characteristics: First, launch a call in a post bar with 30 million attention, quickly connect different circles, and gather a large number of people; second, events often start from micro blog, ferment in cyberspace, act on grassroots groups, which is a bottom-up national reproduction mode. Third, the time from the fermentation period to the outbreak period of collective action is very short, often in 1-2 months; third, the action has a clear division of labor and strict discipline; fourth, the task can be completed in a few days with "flash" action. The above characteristics prove that the Internet has shown a strong function in promoting the key factors of group action, such as spatial aggregation, group consensus, and the production of group unity. As a whole, cyberspace has become a new field for the reproduction of production relations of national community consciousness. 


\section{DIVERSIFIED NETWORK EXPRESSION OF COMMUNITY CONSCIOUSNESS}

Through the continuous construction of field structure and discourse network, the media shapes the expression texture of the real society. According to Sapir Wolff's hypothesis, language in turn affects and shapes the way we perceive and think about the world. In the era of mass media, people's expression of national events is serious and intense, which is closely related to the status and discourse power of the country in the world pattern, as well as the elitist mode of production. Different from the mass media, the Internet's characteristics of empowerment, anonymity, decentralization, interactivity and inclusiveness enrich people's communication methods and create grassroots discourse expression.

Emoticon pack, a kind of expression symbol that came into being with the emergence of the Internet, includes static pictures, GIF dynamic pictures, expressions, actions and words and other forms. It has developed from the period of 1.0 to today, and has been used in Micro-blog and We-chat as version 3.0. ${ }^{1}$ Because of its grassroots, metaphorical and vivid characteristics, it has become the main symbol form of social communication and game communication among more and more youth groups.

On the one hand, emoticon pack is produced on grassroots social platforms, which are rich in the structure and characteristics of general public discourse. In terms of the expression of the consciousness of national community, this kind of discourse, which has been integrated into "collage" and "isomorphism", has formed a sharp contrast with the traditional narrative way: the viewpoint and attitude are clear at a glance; the intention is clear; meanwhile, it has more impact and appeal. On the other hand, the metaphorical features of emoticon pack not only avoid more supervision and are easy to spread and become popular, but also trigger people's potential cognitive structure to express empathy. "Metaphor is a way for us to connect two different areas of experience in an instant, so as to form an approach of illustrative, picturesque, highly general images". Compared with the expression of words, the expression of expression pack is humorous, witty and entertaining. It is often named after other things. Therefore, it gives users a certain sense of security and becomes the carrier of self-expression and emotional release of more and more Internet users.

Looking back at the event of "Emperor bar expedition to FB" in 2016, the expression pack became such a weapon of expression. In the event, the "emperor bar" organization unified the provision and large-scale production of most of the expression packs used in the "expedition", and also integrated some of the expression packs personalized and reconstructed by Internet users, integrating many political, cultural and economic factors, in order to achieve the purpose of transferring value and emotional exchange. It

The 1.0 stage of expression dates back to September 19, 1982, when Farman, a teacher at Carnegie Mellon University, first created the smile symbol ": -)"; the following stage of expression 2.0 is a emoji expression that comes with various social software; and the stage of expression 3.0 is a meme expression created by netizens. should be noted that, in contrast to the past radical expressions based on resentment, emoticon pack add relatively soft discourse features such as satire, banter and self-mockery. As an important form of emotional communication and expression, it directly reflects the user's choice tendency and meaning expression, and reflects the social mentality of this group.

\section{CONCLUSION}

By combing and analyzing the formation, convergence and catalysis, and discourse expression of community consciousness in the light of "emperor BA's expedition", this paper attempts to show that the role of communication media and national community consciousness does not stop at simple, one-way instrumental connection and transmission. In the ideological trend and action of the national community consciousness, the media has gradually changed from an "outsider" to an actor's identity. Just like Latour' $\mathrm{s}$ description of the relationship between society and Science in the theory of actor network, which goes against the subjective and objective, all of them form a network of thoughts and actions that are constructed and evolved together by linking as the subject or the actor in the action.

In addition, the media environment gradually constructed by network media extends the field of human cognition and action. As Post said, it should not be confined to its basic tool attributes, but should be aware of the environment it produces and creates, which has a subtle impact on the change of human life and behavior. This is a kind of communication epistemology that is different from the concept of tools - Media construction concept.

Throughout human history, the constantly evolving media has leapt from a marginal role in the past to a dominant force. As the fundamental pillar of society, mankind needs to maintain a clear and fearless critical consciousness, and more needs to discover the basic values of human beings through phenomena. In the typical case of the "Emperor's Expedition", we can gain insight into the sentiments of the post-90s group to the nation-state. Through their playful expression, they realize that the consciousness of the nation-state community is always through the main line. This spontaneous association of the self with the nationstate and the spirit of destiny is worthy of recognition, especially in the transition period of today's Chinese society. Although the consciousness of the national community is derived from various forms under the influence of the media, it has various defects, but it is the main source of national cohesion and unity. Of course, we also need to worry about the expression of community consciousness in the network background. Under the background of the increasingly strong media technology, will the theme of cyber nationalism be weakened by the power of the media? Will the fundamental orientation of group action be diverted by the control and influence of the media? These thoughts are worthy of further discussion. 


\section{REFERENCES}

[1] Manuel .Castel, The Power of Identity, translated by Cao Rongxiang, social sciences literature press, 2006, p. 54. (in Chinese)

[2] Duara, P. DE-constructing the Chinese nation. The Australian Journal of Chinese Affairs, 1993。

[3] Marx: Complete Works of Marx and Engels, vol. III, People's Publishing House, 1960, p. 25. (in Chinese)

[4] The 41st Statistical Report on the Development of China's Internet, China Internet Information Center (CNNIC), December 2017. (in Chinese)

[5] Li Mujin, "Network Nationalism Opens a New Chapter of Chinese Nationalism", in the International Herald Leader, September 23, 2003. (in Chinese)

[6] Yan Qiang: Patriotic Little Pink, Fan War, and Celestialism Saiboge, Terminal Media, https: / / theinitium. com /article /20160722 opinion- yanqiang- pink- cyborg /. (in Chinese)

[7] Wang Hongzhe, Li Simin, Wu Jing: From "Mi Mei" to "Little Pink": A Study on the Production and Mobilization Mechanism of National Identity in the Context of New Media Business Culture, International Press, 2016,38 (11): 43. (in Chinese)

[8] Elster Geilner, Nationality and Nationalism, translated by Han Hong, Central Compilation and Translation Press, 2002. (in Chinese)

[9] Liu Hailong: Patriotism Like Loving Beans: the Birth of New Media and "Fan Nationalism", Modern Communication, 2017. (in Chinese) 